\title{
First-principles study of ultrafast and nonlinear optical properties of graphite thin films
}

\author{
Mitsuharu Uemoto \\ Department of Electrical and Electronic Engineering, Kobe University, Kobe 657-8501, Japar" \\ Shintaro Kurata \\ Physical Engineering Group, Technology Platform Center, \\ Technology 85 Intelligence Integration, IHI Corporation, Yokohama 235-8501, Japan \\ Norihito Kawaguchi \\ Collaboration \&s Marketing Group, Technology Planning Department, \\ Technology 83 Intelligence Integration, IHI Corporation, Yokohama 235-8501, Japan \\ Kazuhiro Yabana \\ Center for Computational Sciences, University of Tsukuba, 1-1-1 Tennodai, Tsukuba, 305-8577, Japan
}

(Dated: January 12, 2021)

\begin{abstract}
We theoretically investigate ultrafast and nonlinear optical properties of graphite thin films based on first-principles time-dependent density functional theory. We first calculate electron dynamics in a unit cell of graphite under a strong pulsed electric field and explore the transient optical properties of graphite. It is shown that the optical response of graphite shows a sudden change from conducting to insulating phase at a certain intensity range of the applied electric field. It also appears as a saturable absorption, the saturation in the energy transfer from the electric field to electrons. We next investigate a light propagation in graphite thin films by solving coupled dynamics of the electrons and the electromagnetic fields simultaneously. It is observed that the saturable absorption manifests in the propagation with small attenuation in the spatial region where the electric field amplitude is about $4 \sim 7 \times 10^{-2} \mathrm{~V} / \AA$.
\end{abstract}

\section{INTRODUCTION}

Owing to rapid progresses of laser technologies, it has become possible to utilize a pulsed light freely selecting its intensity and duration [1. As for the duration of laser pulses, it has become possible to produce light pulses as short as a few tens of attosecond [2]. Using intense and ultrashort laser pulses, there have been observed a number of intriguing phenomena in solids. For example, high harmonic generation from solids has been extensively explored in the last decade [3]. An induced electric current in glass by the ultrashort pulsed light has been observed, indicating that the insulator can be changed into a conducting material in a very short time-scale [4. Using further intense laser pulses, nonthermal laser processing is expected as an efficient means of micro-fabrication of materials [5].

The present paper aims to report a theoretical and computational analysis on the ultrafast and nonlinear optical responses of graphite thin films. Graphite is a semimetallic layered material of carbon atoms arranged in a honeycomb lattice. Monolayered carbon structures such as graphene and single-wall carbon nanotube have been attracting enormous attention for their unusual optical properteis [6, 7]. For example, large optical nonlinearities such as harmonic generation [8], Kerr effects [9, 10]

\footnotetext{
*uemoto@eedept.kobe-u.ac.jp
}

have been reported. In particular, ultrafast saturable absorption has been extensively investigated and utilized as satulable absorber in a mode-locked fiber laser [1120. Using further intense laser pulses, laser processing of carbon materials has also attracted attention [21, 22]. Since it has been known that micro-fabrication of some carbon materials are difficult by ordinary methods [23, efficient processing using ultrashort pulsed light is highly expected.

To obtain reliable understanding for the ultrafast and nonlinear optical properties, it is essential to describe microscopic electron motion under an optical field. Optical properties of graphenes are characterized by the presence of the Dirac cone in the energy band. Although the valence and the conduction bands slightly overlap in the graphite making it semi-metal, optical properties of graphite are also characterized mostly by the Dirac cone like structure. There have been reported a number of theoretical analyses treating motion of electrons in the Dirac cone. They include analytical modeling and analysis [2426., quasi-classical kinetic theory [27, time-dependent solution for the optical Bloch equation 28, 29, and time-dependent solution for the density matrix equation [9, 30, 31]. There have been carried out first-principles calculations including all valence electrons for nonlinear optical properties in frequency domain [32, 33. Descriptions of nonlinear light propagation is another important issue to understand optical responses of multilayer graphenes and graphite thin films, and also laser processing of carbon materials. There have been used a transfer 
matrix method [34]. Nonlinear propagation method was also developed [35].

In the present study, we will employ a first-principles computational approach based on time-dependent density functional theory (TDDFT) [36, 37. The TDDFT has been known as a useful method to investigate optical properties of molecules and solids with a reasonable accuracy for a moderate computational cost. Solving the time-dependent Kohn-Sham (TDKS) equation in time domain [38, 39, it is possible to describe ultrafast and nonlinear electronic dynamics in matters. Applications of such approaches include nonlinear optical constants [40, high harmonic generations [14], light-matter energy transfer [44, 45].

Solving the TDKS equation in time domain, it is possible to investigate ultrafast electron dynamics in graphite induced by a pulsed electric field without any perturbative approximations. We can describe dynamics of whole $s p$ valence electrons including the dynamics at the Dirac cone. Using ordinary functionals, however, collisional effects cannot be included sufficiently, although carrier relaxation dynamics is known to be important in graphite [46] and graphene [47. This limits the validity of the TDDFT approach to a short duration before the relaxation becomes significant. The relaxation time should depend on the laser parameters and is considered about a few tens of femtosecond [9, 26, 46, 47.

A theoretical description of the propagation of light is another important subject. There has been a progress to develop combined simulations of TDDFT for electron dynamics and electromagnetism analysis for a light propagation adopting a multiscale strategy [48. The method has been successfully applied to analyze ultrafast and nonlinear light propagations: the attosecond transient absorption spectroscopy mimicking pump-probe experiments [49] and the spatial distribution of energy deposition that is the basic information to analyze laser processing [50, 51].

This paper is organized as follows: In Sec. II] we provide a formalism and a computational method based on TDDFT. Electron dynamics in a unit cell of graphite induced by pulsed electric fields are discussed in Sec. III. Light propagations through graphite thin films are discussed in Sec. IV Finally, in Sec. V] a summary will be presented.

\section{THEORY}

\section{A. Electron dynamics in a unit cell}

We describe a time evolution of electron orbitals in a unit cell of graphite under a strong pulsed electric field by solving the TDKS equation for Bloch orbitals. Expressing the applied electric field using a vector potential
$\mathbf{A}(t)$ in the velocity gauge 39, we have:

$$
i \frac{\partial}{\partial t} u_{b \mathbf{k}}(\mathbf{r} ; t)=H[\mathbf{A}] u_{b \mathbf{k}}(\mathbf{r} ; t),
$$

where $u_{b \mathbf{k}}$ is the Bloch orbitals with band index $b$ and wavenumber k. The Hamiltonian $H[\mathbf{A}]$ is given by

$$
\begin{aligned}
H[\mathbf{A}]= & \frac{1}{2 m}\left[\hat{\mathbf{p}}+\hbar \mathbf{k}+\frac{\mathbf{A}(t)}{c}\right]^{2} \\
& +V_{\mathrm{ps}}(\mathbf{r})+V_{\mathrm{H}}[n](\mathbf{r})+V_{\mathrm{xc}}[n](\mathbf{r}),
\end{aligned}
$$

where $V_{\mathrm{ps}}, V_{\mathrm{H}}$ and $V_{\mathrm{xc}}$ are ionic pseudopotentials, Hartree and exchange-correlation potentials, respectively. The Hartree and the exchange-correlation potentials depend on the electron density $n(\mathbf{r} ; t)$ that is expressed as:

$$
n(\mathbf{r} ; t)=\frac{2}{\Omega_{\mathrm{BZ}}} \int_{\Omega_{\mathrm{BZ}}} \mathrm{d} \mathbf{k}^{\prime} \sum_{b}^{(\text {occ })}\left|u_{b \mathbf{k}^{\prime}}(\mathbf{r} ; t)\right|^{2},
$$

where $\Omega_{\mathrm{BZ}}$ is the volume of the Brillouin zone (BZ). The summation of $b$ is taken over all occupied bands. The integration in the BZ is carried out by numerical quadrature using appropriately selected sampling points. Therefore, $\mathrm{Eq}(3)$ can be rewritten as

$$
n(\mathbf{r} ; t)=2 \sum_{\mathbf{k}_{i}}^{B Z} \sum_{b}^{(\text {occ })} w_{i}\left|u_{b \mathbf{k}_{i}}(\mathbf{r} ; t)\right|^{2},
$$

where $\mathbf{k}_{i}$ and $w_{i}$ are the sampled $k$-points and their weighting coefficients, respectively. A uniformly distributed $\mathbf{k}_{i}$ with equal $w_{i}$ are often used for calculations of periodic crystals. In this study, however, we use a nonuniform $k$-point sampling in the BZ to obtain convergent results with less computational costs. Details will be explained in Sec. IIC.

From the Bloch orbitals, the electric current density averaged over the unit cell, $\mathbf{J}(t)$, is given as below:

$$
\begin{aligned}
\mathbf{J}[\mathbf{A}](t)= & -\frac{2}{\Omega_{\text {cell }}} \sum_{b \mathbf{k}_{i}} w_{i} \int_{\Omega_{\text {cell }}} \mathrm{d} \mathbf{r}^{\prime} u_{b \mathbf{k}_{i}}^{\star}\left(\mathbf{r}^{\prime}, t\right) \\
& \left(\hat{\mathbf{p}}+\mathbf{k}+\frac{\mathbf{A}(t)}{c}-i\left[\hat{\mathbf{r}}, V_{\mathrm{NL}}\right]\right) u_{b \mathbf{k}_{i}}\left(\mathbf{r}^{\prime}, t\right),
\end{aligned}
$$

where $V_{\mathrm{NL}}$ is the nonlocal part of the pseudopotential $V_{\mathrm{ps}}$, and $\Omega_{\text {cell }}$ is the volume of the unit cell.

\section{B. Light propagation}

We next consider a description of light propagation in a thin film of graphite. We will use "Maxwell+TDDFT multiscale method" [48, calculating light propagations in solids by combining electromagnetics field analysis and electronic dynamics calculations. We note the the spatial scale of the electromagnetic fields of the propagating light 
is a few hundreds of nanometers, and is much larger than that of the microscopic electronic dynamics. In order to overcome the mismatch of the two spatial scales, we utilize a multiscale method introducing the two coordinate systems for the macroscopic and microscopic dynamics.

The multiscale method is a natural extension of a macroscopic electromagnetism that is usually used to describe light propagation in a medium. In the macroscopic electromagnetism, one usually solve macroscopic Maxwell equation using a grid system in which the grid spacing is typically much larger than atomic scale. The microscopic dynamics is taken into account through a constitutive relation that relates, for example, the electric field and the electric current locally, $J(t)=J[E(t)]$. To describe a propagation of an intense pulsed light, we cannot use any perturbative constitutive relations. Instead, we solve the TDKS equation to relate the current density and the electric field. It is noted that we need to consider electron dynamics at each grid point that is used to solve the macroscopic Maxwell equation, since electron dynamics at each point is different from each other. The microscopic coordinate is used to solve the TDKS equation to calculate microscopic electron dynamics.

For the macroscopic electromagnetic fields, we solve the one-dimensional Maxwell's equation:

$$
\frac{1}{c^{2}} \frac{\partial^{2} A_{X}(t)}{\partial t^{2}}-\frac{\partial^{2} A_{X}(t)}{\partial X^{2}}=\frac{4 \pi}{c} J_{X}(t),
$$

where $X$ is the macroscopic coordinate variable, $A_{X}(t)$ is the vector potential field at $X$. At each point $X$, we consider a microscopic electronic system. At the microscopic scale, we consider an electron dynamics of infinitely extended system under a spatially uniform electric field specified by $A_{X}(t)$ where we treat $X$ as a parameter. The electron motion is described by Bloch orbitals, $u_{X b \mathbf{k}}$, that satisfy

$$
i \frac{\partial}{\partial t} u_{X b \mathbf{k}}(\mathbf{r} ; t)=H\left[A_{X}\right] u_{X b \mathbf{k}}(\mathbf{r} ; t),
$$

The current density $J_{X}(t)$ is determined by Eq. (5) with $J_{X}(t)=J\left[A_{X}\right](t)$. We solve the TDKS and the Maxwell equations simultaneously exchanging $A_{X}(t)$ and $J_{X}(t)$ at every time step.

It should be noted that the present multiscale formalism returns to the ordinary macroscopic electromagnetism when a field is sufficiently weak. The detail of our formalism is explained in the reference [48].

\section{Computational details}

We consider an ABA-stacked graphite crystal. Fig. 11(a) illustrates the crystal structure. The lattice constant and the interlayer distance are set as 1.42 and $3.35 \AA$, respectively. We use a rectangular unit cell of $6.70 \times 4.25 \times 2.46 \AA$, which contains 8 carbon atoms. For electron-ion interaction, we employ norm-conserving (a)

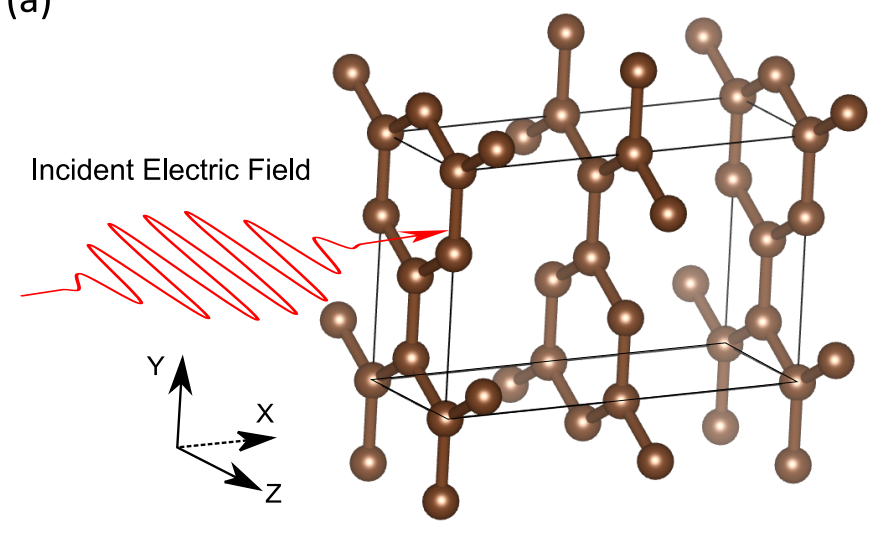

(b)

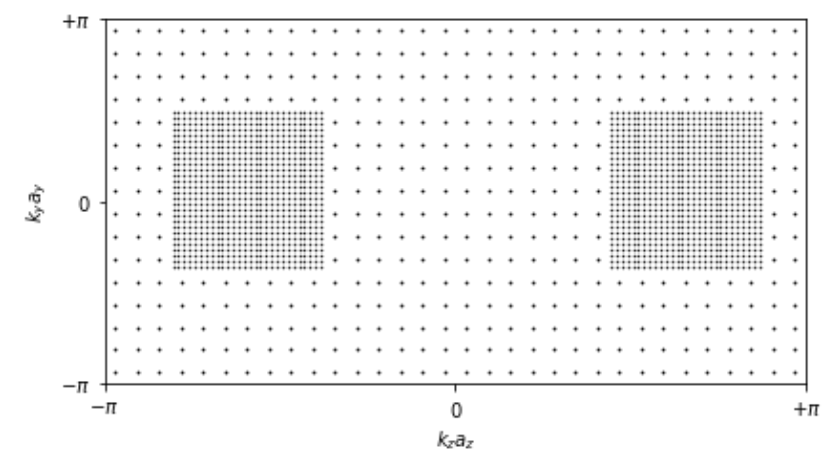

(c)

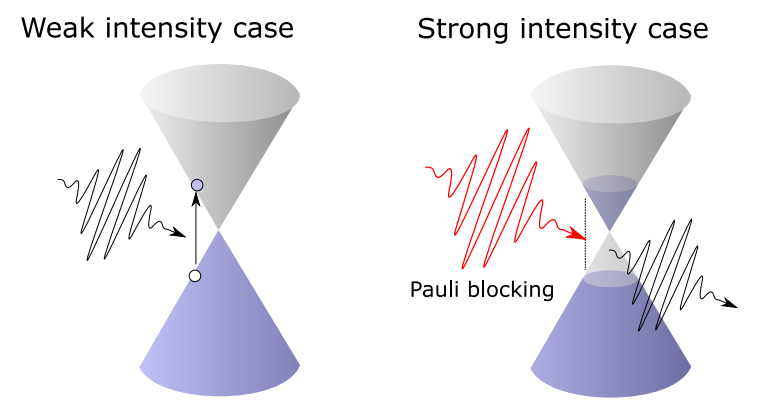

FIG. 1. Schematic illustration of a calculated system: (a) Linearly polarized electric filed irradiates normaly on an ABA-stacked graphite crystal for which we use a rectangular unit cell with 8 carbon atoms. (b) Non-uniform $k$-point distribution to sample the entire BZ that contains 1,656 coarse points and 6,272 dense points. (c) Band structure and electron occupation in the weak (left) and strong (right) field cases.

pseudopotential 52 having 4 valence electrons in a single atom. For the exchange-correlation potential, the adiabatic local density approximation (ALDA) with PerdewZunger functional [53] is adopted. To express Bloch orbitals, we use a three-dimensional Cartesian grid representation with a finite-difference scheme for differentiation operators. The crystalline unit cell is divided into $26 \times 16 \times 16$ uniform grids. The resulting grid spacing is about $0.26 \times 0.27 \times 0.15 \AA$; the grid spacing for $z$-direction ( $E$-field direction) is set to be finer than the other spa- 
tial axes. For the summation over $k$-space in Eqs. (4)(5), the entire BZ is sampled by 7,928 non-uniformly generated $k$-points [see Fig. 11(b)]. Since electronic excitations dominate around the Dirac cone region in the $k$-space [see Fig. 1(c)], we use a non-uniform sampling, a dense sampling for focused regions and a corse sampling in the other areas, to improve the accuracy while avoiding the increase of the computational cost. In the $k_{x}$ (interlayer) direction, the BZ is divided into four. Each $k_{y} k_{z}$-layer is sampled by 414 course points and 1,568 dense points as shown in Fig. 1(b). The weighting coefficients are about $w_{i} \approx 4.88 \times 10^{-4}$ and $3.05 \times 10^{-5}$, respectively. The time evolution is calculated using an enforced time reversal symmetry (ETRS) [54 propagator with a time step of $\Delta t=0.04 \mathrm{au}(0.96 \mathrm{as})$. For computation, we use an open-source TDDFT program package, SALMON (Scalable Ab-initio Light-Matter simulator for Optics and Nanoscience) which has been developed in our group [55].

\section{ELECTRON DYNAMICS IN A UNIT CELL}

\section{A. Dielectric function}

Before discussing nonlinear and ultrafast responses, we first show calculation of the dielectric function of graphite to confirm the reliability of our model in the linear response. We utilize a real-time scheme to calculate the dielectric function as described below 48. Here, as a vector potential in Eqs. (2) and (5), we adopt a Heaviside step function with a small amplitude $\delta A$ :

$$
A_{z}(t)=\delta A \theta(t),
$$

which corresponds to an impulsive electric field described by the Dirac delta function given as $\mathbf{E}(t)=-\partial_{t} \mathbf{A}(t) / c$. For this perturbation, we calculate the induced current density (5) and take the Fourier transformation to obtain the conductivity and the dielectric constant as a function of frequency $\omega$ :

$$
\sigma_{z z}(\omega)=\frac{\int \mathrm{d} t J_{z}(t) e^{i \omega t}}{\int \mathrm{d} t E_{z}(t) e^{i \omega t}}
$$

and

$$
\epsilon_{z z}(\omega)=1+\frac{4 \pi i}{\omega} \sigma_{z z}(\omega)
$$

In Fig. 2, we show the calculated dielectric function $\epsilon_{z z}(\omega)$ (blue solid curve). For comparison, we also show the experimental spectra [56, 57] (broken curves). Since we include all valence orbitals, calculated dielectric function shows reasonable agreement with measurements for wide energy region. In later sections, we will mostly discuss the interaction of laser pulses with a central frequency of $\omega_{1} \sim 1.55 \mathrm{eV}$. At this frequency, the dielectric constant is $\epsilon_{\mathrm{TDDFT}}\left(\omega_{1}\right)=5.3+10 i$ in our TDDFT calculation. This is close to the measured value, $\epsilon_{\exp }\left(\omega_{1}\right) \approx 5+9 i[56$. (a)

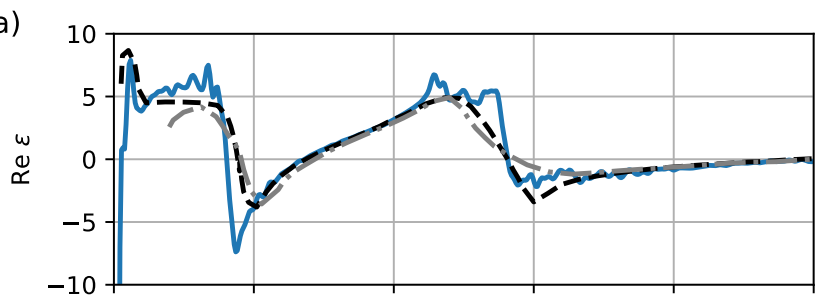

(b)

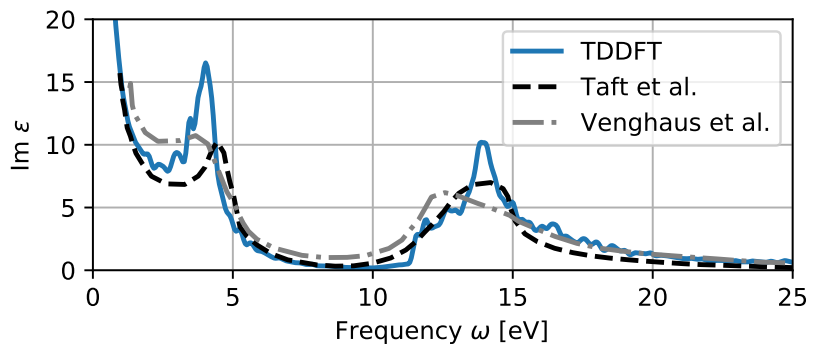

FIG. 2. Dielectric function of a crystalline graphite in the direction parallel to the layer : (a) real part $\operatorname{Re} \varepsilon_{z z}$ and (b) imaginary part $\operatorname{Im} \varepsilon_{z z}$. The experimental spectra are also plotted as black [56] and gray [57] broken curves.

\section{B. Response to pulsed electric fields}

Next, we investigate electronic dynamics and optical responses of graphite induced by a short and strong pulsed electric field. We will use the following waveform:

$$
A_{z}(t)=\frac{E_{\max }}{c \omega_{1}} f(t) \cos \omega_{1} t
$$

with $\cos ^{2}$-type envelope function

$$
f(t)= \begin{cases}{\left[\cos \left(\pi\left(t-T_{P} / 2\right) / T_{P}\right)\right]^{2}} & 0 \leq t \leq T_{P} \\ 0 & \text { otherwise }\end{cases}
$$

where $E_{\max }$ is the maximum amplitude of the electric field and $T_{P}$ is the duration of the pulse envelope. The pulse duration is conventionally expressed using the fullwidth half-maximal (FWHM); for the $\cos ^{2}$-shaped envelope function adopted here, the FWHM duration is given by $T_{\mathrm{FWHM}} \approx 0.364 T_{P}$.

Figure 3 shows (a) temporal profiles of the applied pulsed electric field and (b-e) induced currents for four different amplitudes of the electric field: $E_{\max }=8.7 \times$ $10^{-3} \sim 2.7 \times 10^{-1} \mathrm{~V} / \AA$. The pulse duration is taken to be $T_{P}=80 \mathrm{fs}\left(T_{\mathrm{FWHM}} \approx 30 \mathrm{fs}\right)$. The purple solid curve shows the current density calculated by Eq. (5), and the dotted black curve shows the current density assuming a linear response, using a conductivity: $J_{z}^{(L R)}(t)=$ $\left[\operatorname{Re} \sigma\left(\omega_{1}\right)\right] E_{z}(t)-\left[\operatorname{Im} \sigma\left(\omega_{1}\right) / \omega_{1}\right] \dot{E}_{z}(t)$ with the conductivity from the TDDFT calculation, $\sigma=0.047-0.018 i \mathrm{au}$.

From the calculation, we find the following nonlinear behavior as the amplitude $E_{\max }$ increases. When the applied field amplitude is weak, $J_{z}(t)$ agrees well with the estimate assuming the linear response, $J_{z}^{(L R)}(t)$, as 
(a)

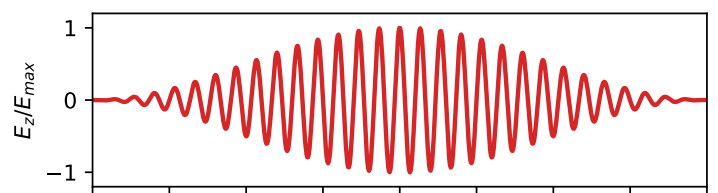

(b)

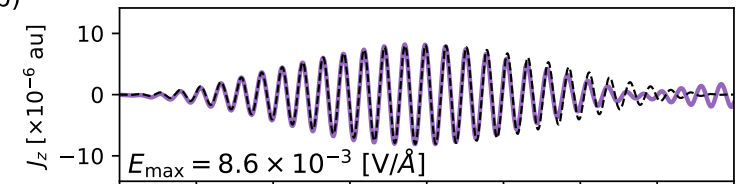

(c)

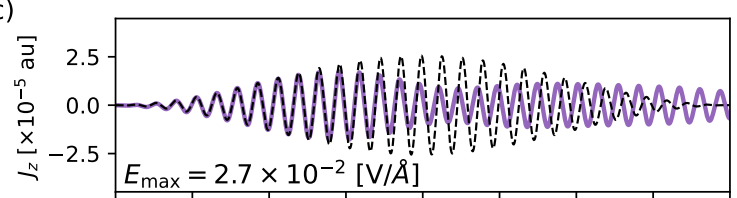

(d)

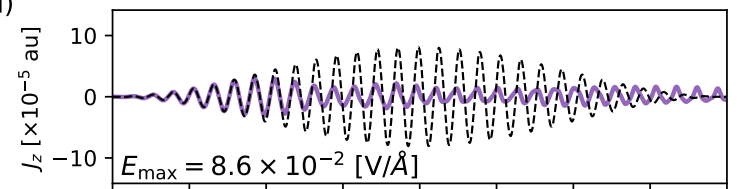

(e)

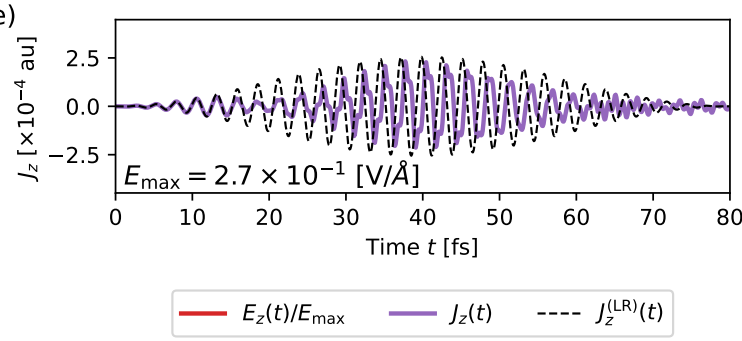

FIG. 3. Applied electric field (a) and induced current density (b-e) for four different maximal field amplitudes $E_{\max }$. The central frequency and the pulse duration are set to $\omega_{1}=1.55 \mathrm{eV}$ and $T_{P}=80 \mathrm{fs}$, respectively. In $(b-e)$, purple lines show calculated current density using Eq. (5). Black broken-line shows the linear results with $\sigma=0.047-0.018 i \mathrm{au}$.

seen in (b) at $E_{\max }=8.6 \times 10^{-3} \mathrm{~V} / \AA$. Here the optical response is conducting: the current is mostly in phase with the electric field, $E(t)$. As the field amplitude increases, the current starts to depart from the linear response. At $E_{\max }=2.7 \times 10^{-2} \mathrm{~V} / \AA$ that is shown in (c), the current gradually attenuates during the pulse irradiation and there also appears a phase change at around $t=40 \mathrm{fs}$. The attenuation becomes maximum in (d) where the field strength is $E_{\max }=8.6 \times 10^{-2} \mathrm{~V} / \AA$. Further increasing the field strength, the amplitude of the current $J_{z}(t)$ increases again in (e) at the field amplitude of $2.7 \times 10^{-1} \mathrm{~V} / \AA$. Here an appearance of high-frequency oscillations is also observed.

We consider that the suppression of the induced current is caused by the saturable absorption (SA). To understand the temporal change of the optical response, it is useful to analyze the relative phase shift between the applied electric field and the induced current, as well as the amplitude. The induced current is in phase with the electric field for conducting case, $J \approx \sigma E$ (conducting (a)

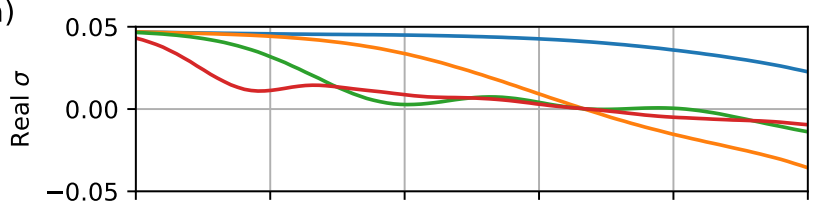

(b)

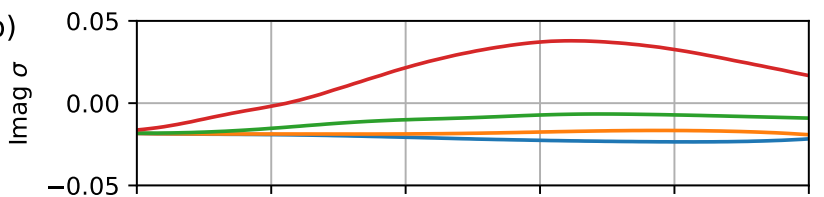

(c)

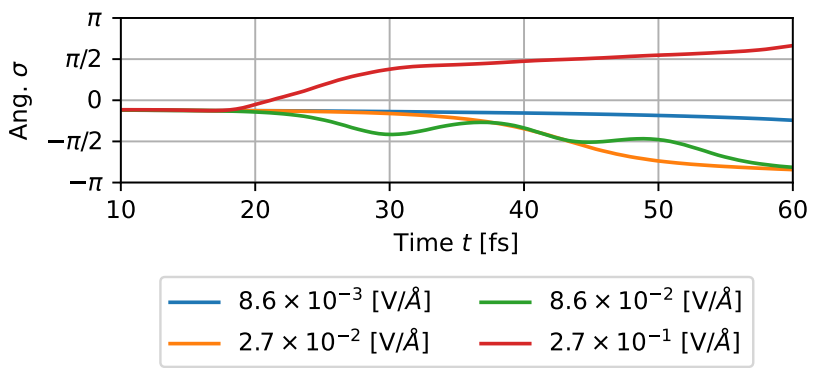

FIG. 4. Temporal-evolution of the complex conductivity calculated from the short-time Fourier transform $\sqrt{13}$ ). The real part (a), imaginary part (b), and phase-angle (c) of the conductivity are plotted for three cases of maximum electric field amplitude.

phase), while the induced current has a phase difference of $\pi / 2$ to the electric field (insulating phase) for insulators, since the current is expressed as the time derivative of the polarization, $J=\partial t P(t)=\chi \partial_{t} E$.

To analyze the phase change quantitatively, we show the short-time Fourier transform:

$$
\sigma(\omega ; t)=\frac{\int J_{z}\left(t^{\prime}\right) e^{i \omega t^{\prime}} w_{b}\left(t^{\prime}-t\right) \mathrm{d} t^{\prime}}{\int E_{z}\left(t^{\prime}\right) e^{i \omega t^{\prime}} w_{b}\left(t^{\prime}-t\right) \mathrm{d} t^{\prime}}
$$

where $w_{b}(t)$ is the Blackman's window function with the width of 10 fs. Figure 4 shows the temporal evolution of the real and imaginary parts, and the phase of the conductivity. At small $E_{\max }$, the conductivity behaves as a constant in time, indicating that the response can be described by the linear response theory. A rather sudden ehange at $t>60 \mathrm{fs}$ is eaused by an artifaet of the short time Fourier transform. As $E_{\max }$ increases, the reduction of the real part $\operatorname{Re} \sigma(\omega ; t)$ and the progression of the phase ang $\sigma(\omega ; t)$ arises. At $E_{\max }=8.6 \times 10^{-2} \mathrm{~V} / \AA$, the phase-difference goes to $\pi / 2$ at the central time of the pulse. These results indicate that the optical property of graphite changes from conductor to insulator under the intense optical field. The red curve in Fig. 4 shows a transient conductivity under a strong applied field corresponding to Fig. 3(e). We find out a characteristic phase inversion in the imaginary part of the conductivity. This change is considered to come from the Drude-like response of metallic media due to an increase of excited 
carriers caused by the strong applied field. We will later discuss the occupation distribution of this case.

Next, we investigate the electronic excitation energy, that is, the energy transfer from the applied electric field to electrons in the unit cell. From the induced current $\mathbf{J}(t)$ under the applied electric field $\mathbf{E}(t)$, the energy deposition per unit time and volume can be evaluated by $W(t)=\mathbf{E}(t) \cdot \mathbf{J}(t)$. Therefore, we introduce the electronic excitation energy per atom at time $t$ by

$$
E_{\text {ex }}(t)=\frac{\Omega_{\text {cell }}}{N_{\text {atom }}} \int_{-\infty}^{t} \mathrm{~d} t^{\prime} \mathbf{E}(t) \cdot \mathbf{J}(t),
$$

where $N_{\text {atom }}$ denotes the number of atoms contained in the unit cell. For a weak field, the excitation energy reduces

$$
E_{\text {ex }}^{(\mathrm{LR})}(t)=\frac{\Omega_{\text {cell }}}{N_{\text {atom }}} \int_{-\infty}^{t} \mathrm{~d} t^{\prime} \operatorname{Re} \sigma_{z z}\left(\omega_{1}\right)\left[E_{z}\left(t^{\prime}\right)\right]^{2},
$$

if the frequency dependence of the conductivity is small.

In Fig. 5, we plot $E_{\text {ex }}(t)$ for four different $E_{\max }$ amplitudes. It shows a clear indication of the SA. In the small amplitude case (a), calculated $E_{\text {ex }}(t)$ (red) behaves close to the linear response result $E_{\mathrm{ex}}^{(\mathrm{LR})}(t)$ (black curve). As the amplitude increases, $E_{\text {ex }}(t)$ greatly departs from $E_{\text {ex }}^{(\mathrm{LR})}(t)$. The ratio of the actual excitation energy to the estimation by the linear response becomes smaller and smaller as the amplitude increases.

According to Eq. 15, $E_{\mathrm{ex}}^{(\mathrm{LR})}(t)$ increases monotonically with $t$. However, in Fig. 5 (b-d), $E_{\text {ex }}(t)$ shows even a descending behavior in the second half of the pulse. This energy reduction is related to the anti-conducting phase shift shown in Fig. 4. The presence of the anti-conducting current $\mathbf{J}(t) \propto-\mathbf{E}(t)$ causes a negative contribution to the energy deposition, $W(t) \leq 0$.

In Fig. 6, we show the total amount of energy transfer from the pulsed electric field to electrons in the unit cell that is equal to the electronic excitation energy after the pulse ends, $E_{\text {ex }}\left(T_{P}\right)$. The energy transfer is plotted against the maximum amplitude of the applied electric field, $E_{\max }$, for four different pulse durations, $T_{P}=10 \mathrm{fs}$, $20 \mathrm{fs}, 40 \mathrm{fs}$, and $80 \mathrm{fs}$. This plot again shows a clear indication of the $\mathrm{SA}$.

At weak amplitude $E_{\max }<10^{-2} \mathrm{~V} / \AA$, the transferred energy is almost quadratic in $E_{\max }$ and is proportional to the pulse duration. These behaviors are consistent with the conducting response. This behavior changes abruptly at and above the amplitude, $E_{\max }=10^{-2} \mathrm{~V} / \AA$. The transferred energy shows a smaller slope or even does not increase for a certain region of the maximum field amplitude in the region of $10^{-2} \sim 10^{-1} \mathrm{~V} / \AA$. At amplitude region of $4 \times 10^{-2} \sim 2 \times 10^{0} \mathrm{~V} / \AA$, the transferred energy is almost independent of the pulse duration $T_{P}$. This indicates that the saturation takes place at an early stage of the irradiation, as quick as $10 \mathrm{fs}$. At amplitude region $E_{\max }>10^{0} \mathrm{~V} / \AA$, the energy transfer again depends on the pulse duration.

\section{Comparison with measurements}

Let us compare the maximum filed amplitude that shows the SA with the measured value of saturation intensity. There are several measurements for single- and multi-layered graphenes. The measured saturation intensity depends strongly on the duration as well as the frequency of the laser pulse. In early measurements, longer pulses of more than picosecond duration was used. Later there has been several measurements that use pulses of femtosecond duration that correspond well with our caulcations.

In our calculation, the saturation starts at the field amplitude of $4 \times 10^{-2} \mathrm{~V} / \AA$ that corresponds to the intensity of the pulse, $I=1.9 \times 10^{10} \mathrm{~W} / \mathrm{cm}^{2}$ if we assume that the maximum field amplitude in the medium is equal to that of the incident pulse. This should be a good approximation for an extremely thin films. We compare our value with measurements that are conducted in similar physical conditions using laser pulses of wavelength of about $800 \mathrm{~nm}$. There are a few measurements using graphite thin films. Using the pulse of 20 fs duration and for 280 layered graphene, $I_{\text {sat }}=3.0 \times 10^{10} \mathrm{~W} / \mathrm{cm}^{2}$ was reported [58]. Using the pulse of 56 fs duration and for 60 layered graphene, $I_{\text {sat }}=5.7 \times 10^{10} \mathrm{~W} / \mathrm{cm}^{2}$ was reported [15. There are also a few measurements for a single layer of graphene. Using the pulse of $80 \mathrm{fs}$ duration, $I_{\text {sat }}=2.3 \times 10^{10} \mathrm{~W} / \mathrm{cm}^{2}$ was reported [12]. Using the pulse of $100 \mathrm{fs}$ duration, $I_{\text {sat }}=7.6 \times 10^{11} \mathrm{~W} / \mathrm{cm}^{2}$ was reported [18. There was also a measurement using the pulse of duration 200 fs giving $I_{\text {sat }}=(4 \pm 1) \times 10^{9}$ $\mathrm{W} / \mathrm{cm}^{2}$ 14. These measurements using light pulses of shorter than a few hundred femtoseconds, the saturation intensity in our calculation coincides more or less with measurements. There are also theoretical calculation: $I_{\text {sat }}=5 \times 10^{9} \sim 1 \times 10^{10} \mathrm{~W} / \mathrm{cm}^{2}$ using tight-binding model [31, and $I_{\text {sat }}=6.5 \times 10^{10} \mathrm{~W} / \mathrm{cm}^{2}$ including various many-body effects [15]. They also show a reasonable agreement although physical effects included are different in each approach.

In early measurements, much lower saturation intensity was reported using lower frequency and longer pulses; for example, $I_{\text {sat }}=7.4 \times 10^{6} \mathrm{~W} / \mathrm{cm}^{2}$ using a laser pulse of wavelength $1550 \mathrm{~nm}$ and duration $3.8 \mathrm{ps} \mathrm{59}$. For longer pulses, it becomes important to take account of equilibrium processes by collisional relaxations.

\section{Occupation distribution}

Next, we discuss how the occupation of electrons changes during the irradiation of the pulse. For this purpose, we perform density of states (DoS) analysis of the excited carriers. We first define the total-DoS (tDoS) 

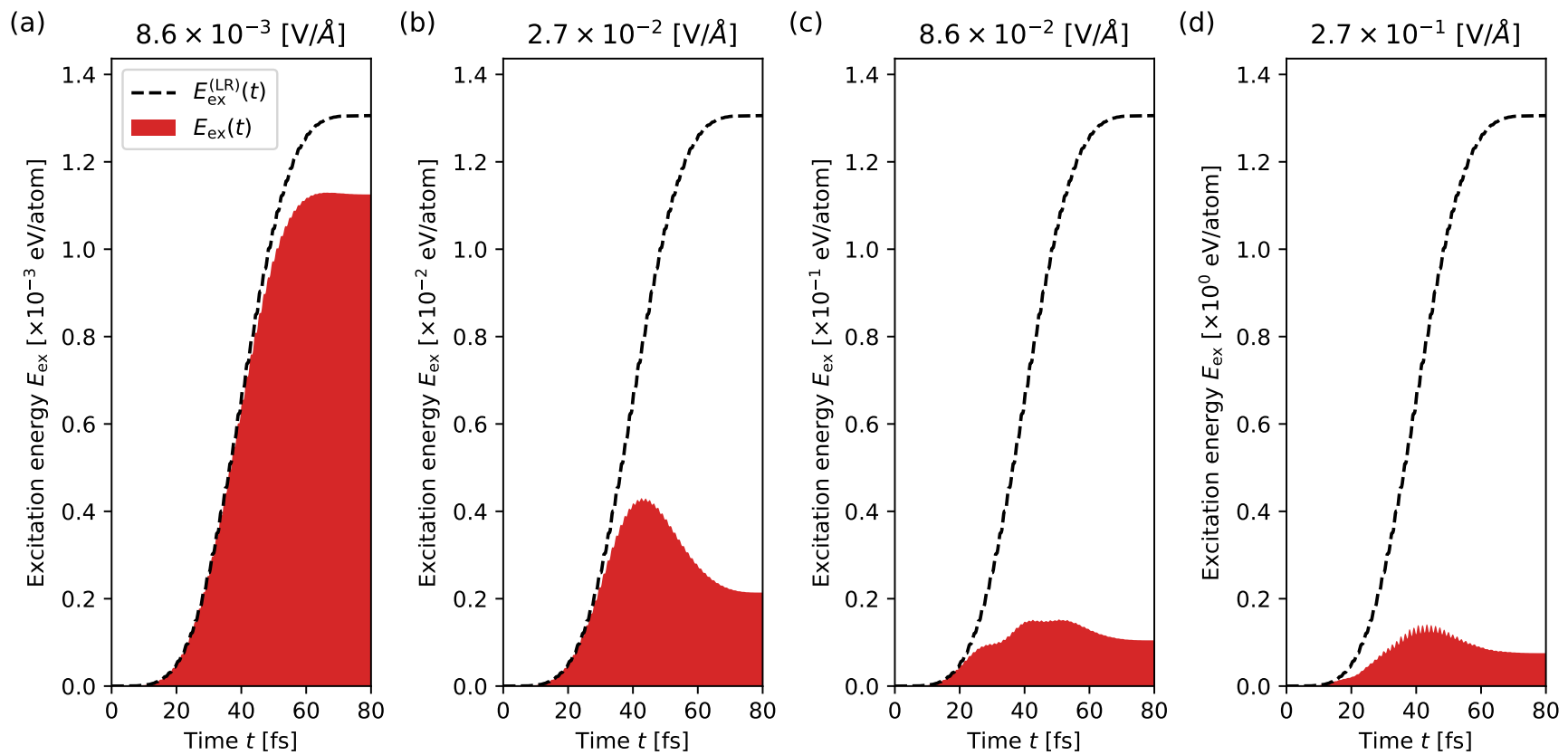

FIG. 5. Excitation energy as a function of time, $E_{\text {ex }}(t)$, for four cases of different field amplitudes. The broken curve is calculated assuming the linear response, $E_{\mathrm{ex}}^{(\mathrm{LR})}(t)$.

$D(E)$ in the ground state by

$$
D(E)=\frac{2}{N_{\text {atom }}} \sum_{b}^{\text {(all) }} \sum_{\mathbf{k}_{i}} w_{i} \delta\left(E-\epsilon_{b \mathbf{k}_{i}}\right)
$$

where $\epsilon_{b \mathbf{k}_{i}}$ is the single particle energy of the orbital $b$ at $\mathbf{k}_{i}$, and the summation is taken over all orbitals. We next define the projected-DoS (pDoS) that indicates the electron occupancy at time $t$,

$$
D^{(\text {proj })}(E)=\frac{2}{N_{\text {atom }}} \sum_{b}^{(\text {all })} \sum_{\mathbf{k}_{i}} w_{i} \delta\left(E-\epsilon_{b \mathbf{k}_{i}}\right) P_{b \mathbf{k}_{i}}(t)
$$

with

$$
P_{b \mathbf{k}}(t)=\sum_{b^{\prime}}^{(\mathrm{occ})}\left|\int_{\Omega_{\text {cell }}} \mathrm{d} \mathbf{r} u_{b^{\prime} \mathbf{k}}^{\star}(\mathbf{r} ; t) u_{b \mathbf{k}+\mathbf{A}(t) / c}(\mathbf{r})\right|^{2},
$$

where we use the so-called Houston function, $u_{b \mathbf{k}+\mathbf{A}(t) / c}(\mathbf{r})$ as the reference state to define the electronic excitation.

Figure 7 shows the tDoS (16) and the pDoS (17). Fig. 7(a)-(c) show the electron occupation at times $t=$ 20,40 , and $60 \mathrm{fs}$ under the field of maximum amplitude $E_{\max }=2.7 \times 10^{-2} \mathrm{~V} / \AA$ that corresponds to Fig. 3(c). Each timing corresponds to (a) linear, (b) saturation, and (c) anti-conducting responses, respectively. In Fig. 7)(a), there appear small peaks of excited electrons and holes at $\approx \pm 0.8 \mathrm{eV}$ from the Fermi surface. The separation of two peaks is equal to the average frequency of the pulse, $\omega_{1}=1.55 \mathrm{eV}$. The maximum density of the excited carriers is two orders of magnitude smaller than the tDoS. At $t=40$ fs shown in Fig. 7(b), the excited carrier density is comparable to the tDoS. At this time, the induced current is substantially suppressed as seen in Fig. 3(c). The occupation change explains the mechanism of the SA: substantial part of the valence electrons are already excited, while the conduction states are mostly filled. These two effects suppress the excitation of electrons. We note that the occupation change is only a fraction of the tDoS as seen in Fig. 7 (b). Since electronic excitations take place anisotropically in $k$-space, the saturation appears although only a fraction of electrons is excited in energy representation. It should, however, noted that the present TDDFT calculation does not take full account of the relaxation effects since the $e-e$ collision effects are not included sufficiently. As noted in the Introduction, thermalization of the electron distribution within a few tens of femtosecond was experimentally reported for graphite [46. At $t=60 \mathrm{fs}$ when the anticonducting response appears in Fig. 33(c), the number of excited carriers becomes small compared with that at the time $t=40 \mathrm{fs}$. It indicates that the appearance of the anti-conducting current is related with the decrease of the carrier density.

Figure 7(d) shows the occupation distribution at the peak time of the field when an intense field of $2.7 \times$ $10^{-1} \mathrm{~V} / \AA$ was applied. The field amplitude corresponds to that used in Fig. 3(e). We can see that the carriers distribute in wide energy. It originates from multiple excitation processes in which excited carriers are re-excited 


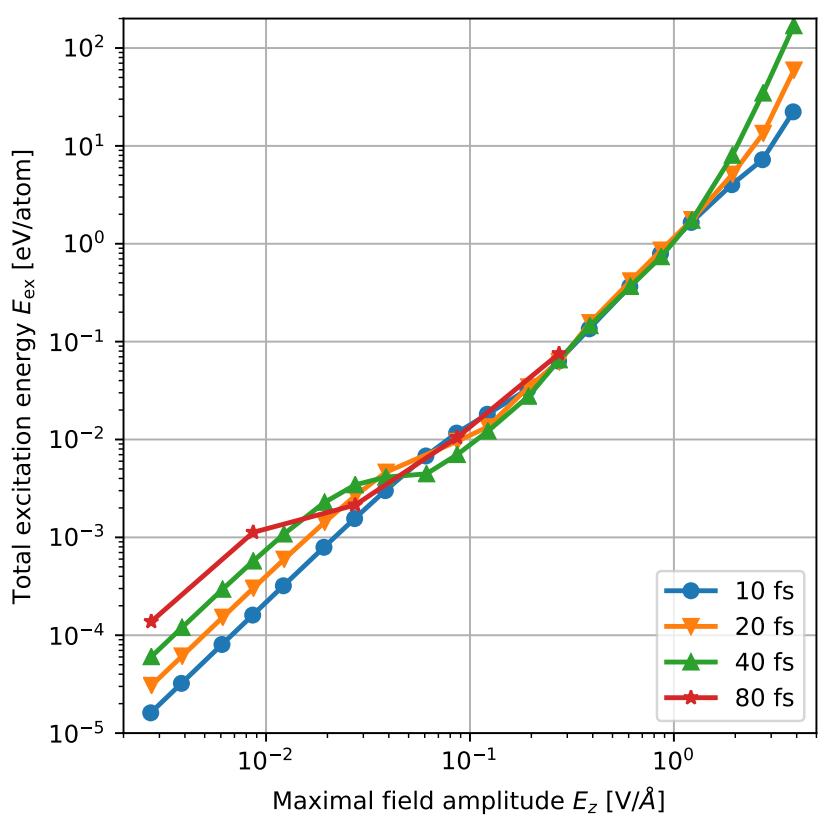

FIG. 6. Energy transfer from the pulse to the medium as a function of the maximum amplitude of the electric field, $E_{\max }$, for four different pulse durations, $T_{P}=10,20,40$ and 80 fs.

by the applied electric field and make transitions to a higher energy band. These final states have a higher DoS than those of Dirac cone and allow existence of high density carriers. This high density carriers are expected to contribute to the metallic response that was observed in Fig. 4. At this field strength, we still observe a strong SA as seen in Fig. 5(d) and Fig. 6.

\section{LIGHT PROPAGATION}

We first summarize a description of the light propagation in ordinary electromagnetism. The reflectance $R$ and the penetration depth $L_{p}$ at the surface of the graphite is given by

$$
R=\frac{(n-1)^{2}+\kappa^{2}}{(n+1)^{2}+\kappa^{2}}, \quad L_{p}=\frac{c}{2 \omega \kappa},
$$

where $n$ and $\kappa$ are the real and the imaginary parts of the index of refraction of graphite, respectively. $\omega$ is the frequency of the light. From the dielectric constant, $\epsilon_{\exp }\left(\omega_{1}\right) \approx 5+9 i[56$, the index of refraction of the graphite is given by $n\left(\omega_{1}\right)+i \kappa\left(\omega_{1}\right)=2.9+1.7 i$ at $\omega_{1}=1.55 \mathrm{eV}$. From these values, we obtain $R=0.36$ and $L_{p}=36 \mathrm{~nm}$, respectively. When a weak light irradiates normally on the bulk graphite surface, the intensity of the pulse decays exponentially as $I \propto \exp \left(-x / L_{p}\right)$ at the penetration distance $x$.

\section{A. Thin film of $50 \mathrm{~nm}$}

In this subsection, we will investigate light propagation through a thin film of graphite of $50 \mathrm{~nm}$ thickness. This corresponds to about 150 sheets of honeycomb layers. Since the thickness is comparable to the absorption depth of the graphite, a part of the incident light will transmit to the opposite side. In the next subsection, we consider a thicker film without transmission.

Utilizing multiscale method described in Sec. IIB, we calculate light propagation by solving the wave equation of Eq. (6) combining electron dynamics described by Eq. (7). We consider a linearly polarized light irradiating normally on the graphite thin film. In Fig. 8, we plot the electric field profiles at several times. The pulsed light initially stays in the left vacuum region as shown in (a), and propagates toward positive $x$-direction. The red line shows the field for the case of a strong pulse with the maximum intensity of $10^{12} \mathrm{~W} / \mathrm{cm}^{2}$ at the vacuum. The black line shows the field for the case of a weak pulse with the maximum intensity of $10^{10} \mathrm{~W} / \mathrm{cm}^{2}$. The black line is multiplied by a factor of 10 so that two curves coincide with each other in the vacuum region. The difference of two curves shows nonlinear effects in the propagation. At the final time shown in (e), we clearly observe a consequence of the SA. Compared with the weak pulse, the transmitted electric field of the strong pulse is much stronger. Looking in detail, the front part of the two pulses coincides with each other. After a few cycle, the electric field of the strong pulse is much larger than the weak pulse. We also find a decrease of the reflected wave for the strong pulse case.

In Fig. 9, we show the intensity(fluence) dependence of the reflectance $R$, the transmittance $T$, and the absorbance $A$ that satisfy $R+T+A=1$. The pulse frequency and duration are set to $1.55 \mathrm{eV}$ and $20 \mathrm{fs}$, respectively. As seen from the figure, nonlinear behavior becomes visible at and above the intensity of $1 \times 10^{11}$ $\mathrm{W} / \mathrm{cm}^{2}$. The transmittance increases as high as 0.4 , about three times larger than the estimate from the linear response. Both the reflectance and the absorbance decrease for the strong field.

In Ref. 58, there was a measurement of transmission of a laser pulse of $775 \mathrm{~nm}$ and $20 \mathrm{fs}$ duration through a multilayer turbostraic graphene of 280 layers. It was reported that the transmission starts to increase at the intensity of $3 \times 10^{9} \mathrm{~W} / \mathrm{cm}^{2}$. The increase of the transmission is about $13 \%$ at the intensity of $3 \times 10^{10} \mathrm{~W} / \mathrm{cm}^{2}$. In Ref. [15], there was a measurement of transmission of a laser pulse of $800 \mathrm{~nm}$ and 56 fs duration through 60 layers film. It was reported that the saturation intensity is $5.7 \times 10^{10} \mathrm{~W} / \mathrm{cm}^{2}$ and the enhancement of $13 \%$ in the transmittance was observed at the intensity of $1.4 \times 10^{11}$ $\mathrm{W} / \mathrm{cm}^{2}$. In view of the difference of the pulse duration and film thickness, our result is in qualitative agreements with these measurements. 
(a)

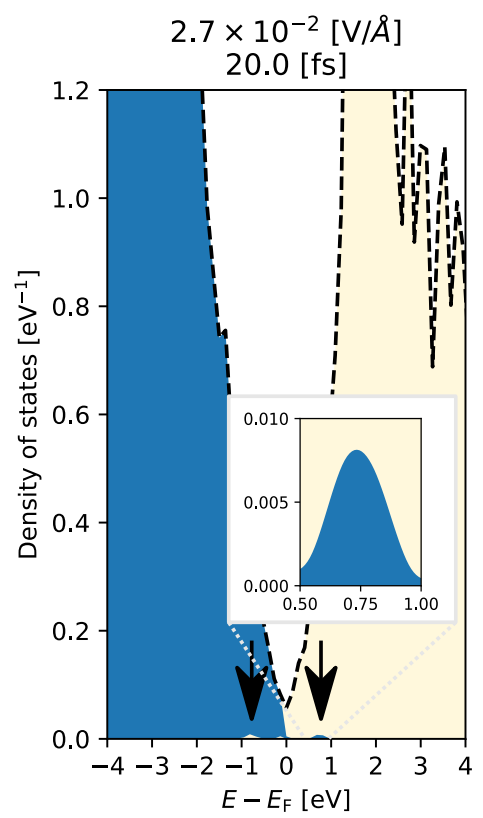

(b)

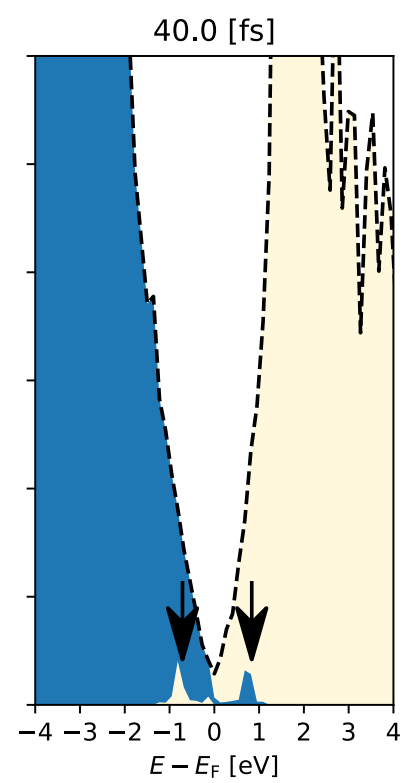

(c)

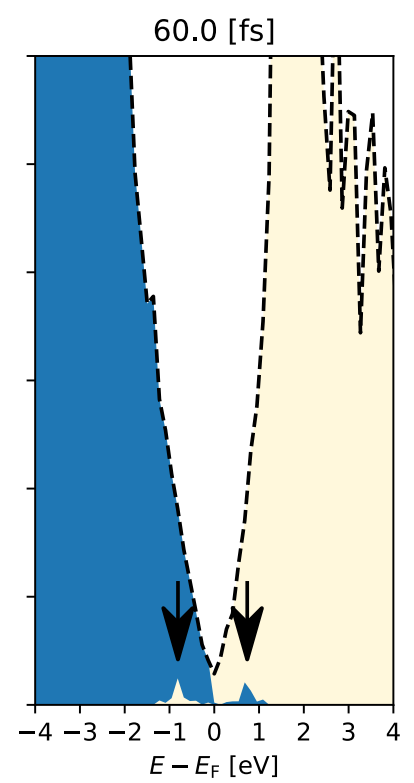

(d)

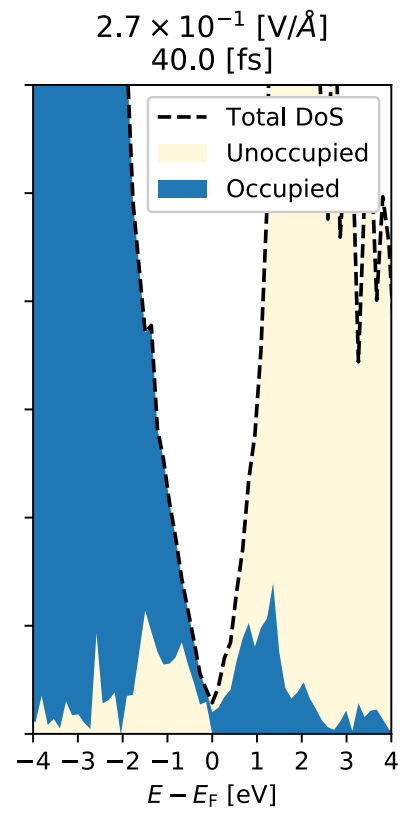

FIG. 7. Temporal-evolution of the pDoS at the vicinity of the Fermi level. The applied pulse duration and maximal field amplitudes are set as $T_{P}=40$ fs and $E_{\max }=2.7 \times 10^{-2} \mathrm{~V} / \AA(\mathrm{a}-\mathrm{c})$ and $2.7 \times 10^{-1} \mathrm{~V} / \AA(\mathrm{d})$, which correspond to Fig. 3 (c) and Fig. 3(e), respectively. The total DoS is plotted as a broken line.

(a)

(b)

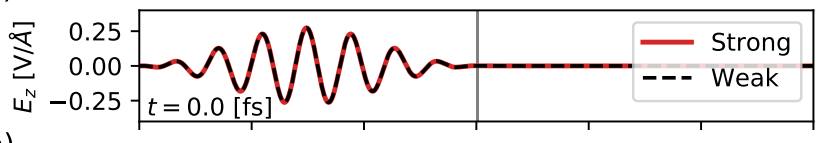

(c)

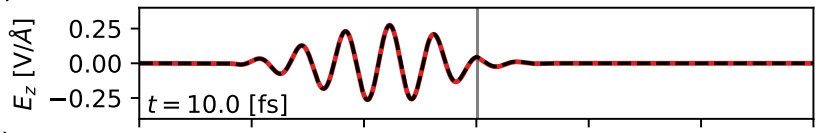

(d)
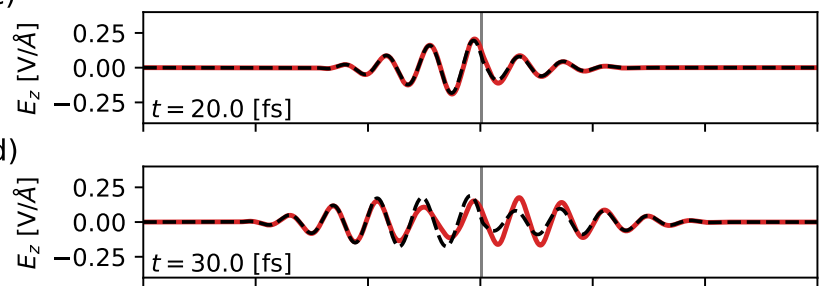

(e)

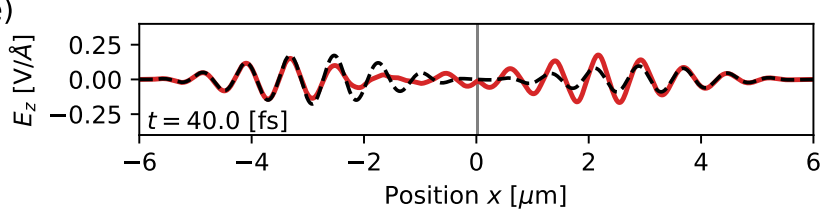

FIG. 8. Time evolution of the electric field of a laser pulse irradiating normally on the graphite thin film of $50 \mathrm{~nm}$ thickness (gray region). Two different intensity cases are compared, the maximum intensity in the vacuum of $I=$ $10^{12} \mathrm{~W} / \mathrm{cm}^{2}$ (red) and $I=10^{10} \mathrm{~W} / \mathrm{cm}^{2}$ (black). The pulse duration is set as $T_{P}=20 \mathrm{fs}$.

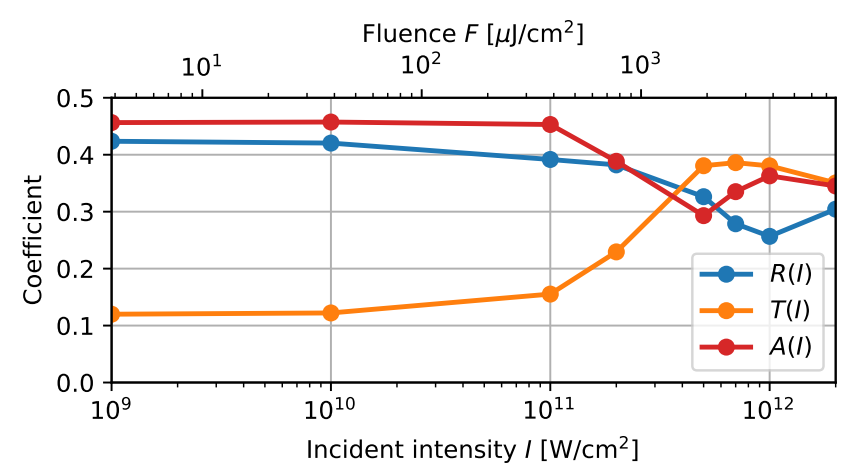

FIG. 9. Intensity-dependence of reflection $R(I)$ and transmittion coefficients $T(I)$ for the thin-film graphite $50 \mathrm{~nm}$ thickness. The pulse duration is set to $T_{P}=20 \mathrm{fs}$.

\section{B. Thicker film of $250 \mathrm{~nm}$}

We next consider the light propagation through a film of $250 \mathrm{~nm}$ thickness. Since it is much thicker than the penetration depth, we expect a very small transmission. We consider again a linearly polarized light normally irradiating on the thin film. The intensity and the duration of the incident pulse is set as $I=10^{12} \mathrm{~W} / \mathrm{cm}^{2}$ and $T_{P}=20 \mathrm{fs}$, respectively.

We show a typical light propagation in Fig. 10. The red line shows the electric field of the propagating light. 
(a)

(b)
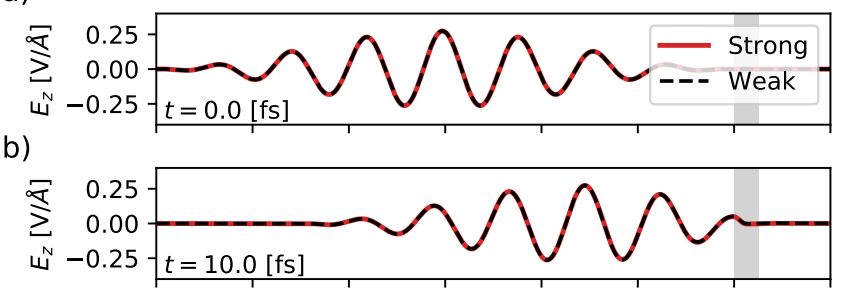

(c)

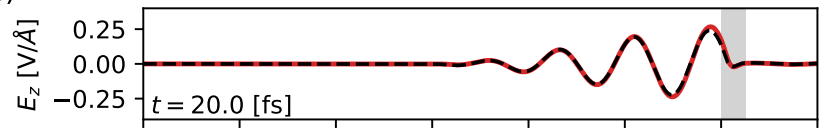

(d)

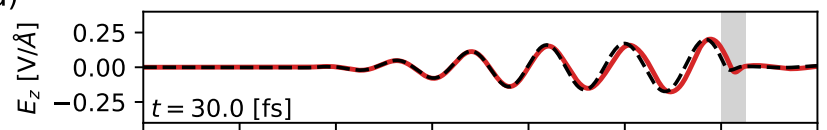

(e)

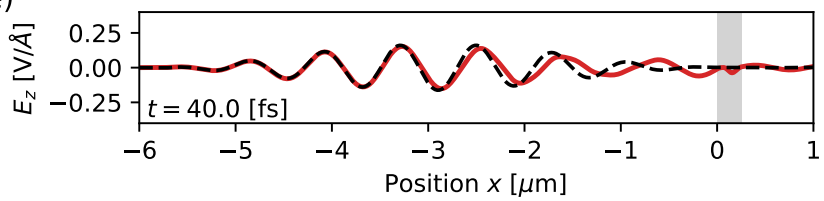

FIG. 10. Time evolution of a pulsed light irradiating on the graphite thin film of $250 \mathrm{~nm}$ thickness (gray region). A red line shows the electric field of a strong pulse with maximum intensity of $I=10^{12} \mathrm{~W} / \mathrm{cm}^{2}$. A dashed black line shows the electric field of a weak pulse magnified so that the incident pulses look the same.

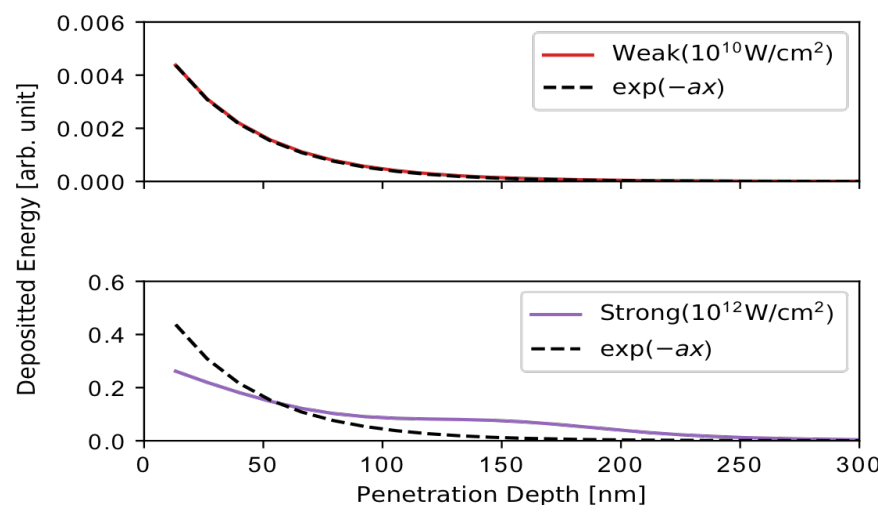

FIG. 11. Energy deposition as a function of distance from the surface. (a) for weak pulse and (b) for strong pulse cases.

As expected, all the pulse is reflected or absorbed at the film. The broken black line shows the propagation of the weak pulse, multiplied with a constant so that it coincides in the linear limit. Looking at the reflected wave of the bottom panel (e), the front part of the electric field of two intensities are close to each other. After $t=10 \mathrm{fs}$, there appears a phase delay in the strong pulse.

In Fig. 11, we show the energy deposition from the laser pulse to electrons in the medium as a function of the distance from the surface, $x$. For comparison, the energy deposition assuming a linear response is shown by the dashed curve. It shows the exponential decay as a function of $x$. In the upper panel, we show the case of the laser pulse of the incident intensity $I=10^{10}$ $\mathrm{W} / \mathrm{cm}^{2}$. In this case, the deposited energy is well fit by the exponential curve, indicating that the absorption can be described by the ordinary ohmic resistance. In the lower panel, the absorption of the laser pulse of the intensity $I=10^{12} \mathrm{~W} / \mathrm{cm}^{2}$ is shown. At the surface, the absorption is weaker than the estimate by the linear response, which is caused by the SA. As the distance from the surface increases, the energy deposition becomes larger than the estimate by the linear response. Because of the SA, the laser pulse is not absorbed efficiently at the surface, and the pulse can reach deeper inside the materials. It then causes the enhanced energy deposition deep inside the medium.

To obtain a systematic understanding, we summarize in Fig. 12(a) the deposited energy $E_{\text {ex }}(x)$ as a function of the penetration depth from the surface $x$, and in Fig. 12(b) the maximum electric field at the depth $x$. They are shown for pulses with different maximum intensities. The pulse duration is chosen to be $T_{P}=20$ fs. In Fig. 12(a), the black dotted line is an exponential curve $\exp \left(-x / L_{P}\right)$ with $L_{P}=36 \mathrm{~nm}$ that is expected in the linear response (19).

At small intensity $I \leq 1 \times 10^{11} \mathrm{~W} / \mathrm{cm}^{2}$, The slope of $E_{\text {ex }}(t)$ is well fit by the exponential function, indicating the linear response. The change in slope around $x=$ $200 \sim 250$ that can be seen at all intensities is due to the reflection at the back-surface $(L=250 \mathrm{~nm})$. At $I=2 \times 10^{11} \mathrm{~W} / \mathrm{cm}^{2}$, the SA becomes appreciable and the slope of $E_{\text {ex }}(x)$ becomes small in the region about $x \leq 50 \mathrm{~nm}$. From Fig. 12(b), the maximum electric field at the surface is about $0.06 \mathrm{~V} / \AA$ for the incident pulse of $I=2 \times 10^{11} \mathrm{~W} / \mathrm{cm}^{2}$. At this field amplitude, a sizable $\mathrm{SA}$ is seen in the single cell calculation as seen in Fig. 6 .

In the range of $5 \times 10^{11} \sim 1 \times 10^{12} \mathrm{~W} / \mathrm{cm}^{2}$, a plateau with a very small gradient of $E_{\mathrm{ex}}(x)$ appears in the vicinity of $x=100 \mathrm{~nm}$ where the maximum electric field amplitude is close to $4 \sim 7 \times 10^{-2} \mathrm{~V} / \AA$. This range of amplitude coincides again with the region where the SA is seen in Fig. 6. The SA makes the pulse penetrate deeper inside the medium than that expected from the linear response. For the incident pulse with $I>5 \times 10^{11} \mathrm{~W} / \mathrm{cm}^{2}$, the energy deposition at the surface increases as the intensity increase, and shows a slope that is smaller than that expected from the linear response. Here the maximum electric field amplitude exceeds $0.1 \mathrm{~V} / \AA$ where, while the SA is still significant, the excitation energy increases as the field amplitude increases as seen in Fig. 6.

In Fig. 12(c), the deposited energy is plotted against the maximum electric field amplitude. This is constructed from the results of (a) and (b), removing the information of the depth $x$. We can indeed confirm that the deposited energy is very well correlated with the local value of the maximum electric field amplitude. We find that the deposited energy is almost the same for a region of electric field amplitude, $0.4 \mathrm{~V} / \AA<E_{\max }<0.8 \mathrm{~V} / \AA$. 
(a)

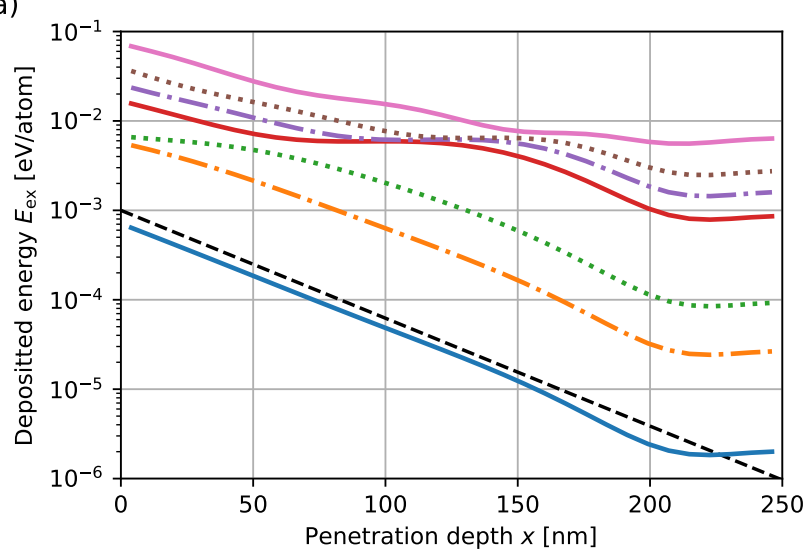

(b)

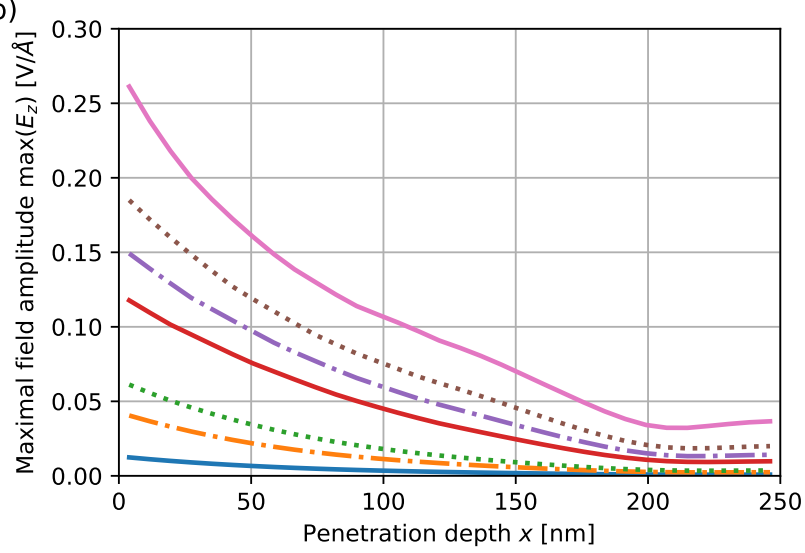

(c)

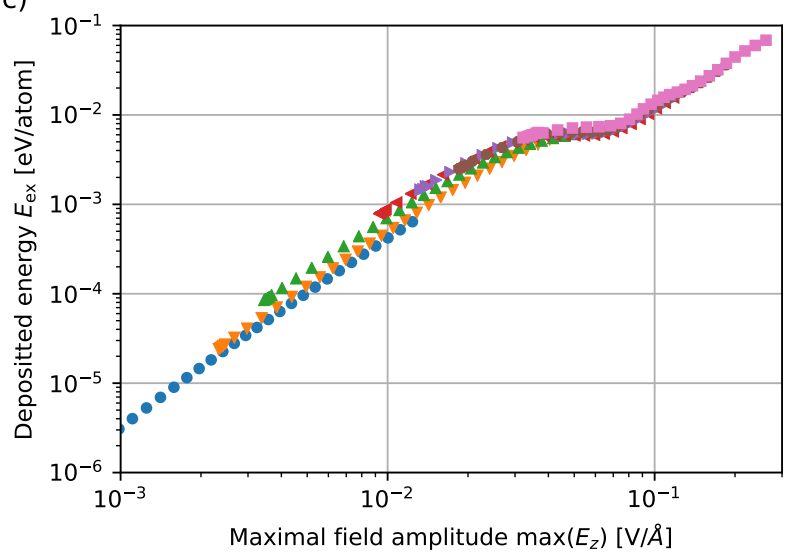

FIG. 12. (a) Deposited energy $E_{\text {ex }}(x)$ in the thin-film graphite of $250 \mathrm{~nm}$ thickness as a function of the distance from the surface $x$, (b) maximum electric field amplitude at the depth $x$, and (c) deposited energy plotted against the maximum electric field amplitude. The curves shown are for pulses of intensities $I=10^{9} \sim 10^{13} \mathrm{~W} / \mathrm{cm} 2$. The pulse duration is set to $T_{P}=20 \mathrm{fs}$ for all intensities.

This coincides accurately with the result shown in Fig. 6 at $T_{P}=20 \mathrm{fs}$.

\section{SUMMARY}

We investigate ultrafast and nonlinear optical responses of graphite thin films employing the firstprinciples TDDFT. First, we investigated optical responses in a unit cell of graphite under a pulsed electric field. We carried out calculations using a pulsed electric field of various maximum amplitude and duration. We find that a SA dominates in the nonlinear response. During the irradiation of a pulsed electric field of a few tens of femtosecond, there appears a change of optical response from conducting, insulating, and anti-conducting phases. The SA becomes significant above a certain threshold of the maximum amplitude of the applied electric field, around $E_{\max } \sim 0.01 \mathrm{~V} / \AA$. The threshold amplitude becomes smaller as the pulse duration increases. The energy transfer from the pulsed electric field to electrons in the medium is found to saturate very quickly, as fast as 10 fs. At sufficiently high amplitude above $E_{\max }=1$ $\mathrm{V} / \AA$, the saturation disappears.

We next carried out calculations of a propagation of pulsed light through thin films of graphite. Coupled multiscale calculations of light propagation and electron motion are carried out, making it possible to investigate the nonlinear light propagation in the first principles level. For a thin film of graphite with $50 \mathrm{~nm}$ thickness that is comparable to the penetration depth, the transmitted wave shows a clear indication of the SA. We investigated the reflectance, transmittance, and absorbance for a pulsed light of 6 fs FWHM duration and various maximum intensities. We have found the effect of the SA becomes substantial at and above the maximum intensity of $I=1 \times 10^{10} \mathrm{~W} / \mathrm{cm}^{2}$. The transmittance increases from 0.12 in the linear response region to 0.4. We also performed a calculation for a thick sample of $250 \mathrm{~nm}$ thickness where transmitted wave is very small. We find that the intense pulse penetrates deeper inside the medium by the SA. Looking in detail the energy deposition from the light pulse to electrons, there appears a plateau region of absorbance for the field amplitude of 
$0.04<E_{\max }<0.07 \mathrm{~V} / \AA$. This makes the penetration of the light pulse deeper inside the medium for pulses of maximum intensity $I>2 \times 10^{11} \mathrm{~W} / \mathrm{cm}^{2}$.

\section{ACKNOWLEDGEMENTS}

We acknowledge the support by MEXT as a priority issue theme 7 to be tackled by using Post-K Computer and JST- CREST under Grant No. JP-MJCR16N5 and by JSPS KAKENHI under Grant Nos. 20H02649 and 20K15194. M.U. also acknowledge Iketani Science and Technology Foundation. Calculations are carried out at Oakforest-PACS at JCAHPC through the Multidisciplinary Cooperative Research Program in CCS, University of Tsukuba, and through the HPCI System Research Project (Project Nos. hp180088 and hp190106).
[1] T. Brabec and F. Krausz, Intense few-cycle laser fields: Frontiers of nonlinear optics, Rev. Mod. Phys. 72, 545 (2000)

[2] F. Krausz and M. Ivanov, Attosecond physics, Rev. Mod. Phys. 81, 163 (2009)

[3] S. Ghimire, A. D. Dichiara, E. Sistrunk, P. Agostini, L. F. Dimauro, and D. A. Reis, Observation of high-order harmonic generation in a bulk crystal, Nature Physics 7, 138 (2011)

[4] A. Schiffrin, T. Paasch-Colberg, N. Karpowicz, V. Apalkov, D. Gerster, S. Mühlbrandt, M. Korbman, J. Reichert, M. Schultze, S. Holzner, J. V. Barth, R. Kienberger, R. Ernstorfer, V. S. Yakovlev, M. I. Stockman, and F. Krausz, Optical-field-induced current in dielectrics., Nature 493, 70 (2013)

[5] B. N. Chichkov, C. Momma, S. Nolte, F. vonAlvensleben, and A. Tunnermann, Femtosecond, picosecond and nanosecond laser ablation of solids, Applied physics. A, Materials science \& processing 63, 109 (1996)

[6] F. Bonaccorso, Z. Sun, T. Hasan, and A. C. Ferrari, Graphene Photonics and Optoelectronics, Nature Photonics 4, 611 (2010), arXiv:1006.4854.

[7] S. Yu, X. Wu, Y. Wang, X. Guo, and L. Tong, 2d materials for optical modulation: Challenges and opportunities, Advanced Materials 29, 1606128 (2017), https://onlinelibrary.wiley.com/doi/pdf/10.1002/adma.201

[8] N. Yoshikawa, T. Tamaya, and K. Tanaka, Highharmonic generation in graphene enhanced by elliptically polarized light excitation, Science 356, 736 (2017), https://science.sciencemag.org/content/356/6339/736.full.pdf

[9] J. L. Cheng, N. Vermeulen, and J. E. Sipe, Third-order nonlinearity of graphene: Effects of phenomenological relaxation and finite temperature, Phys. Rev. B 91, 235320 (2015)

[10] S. Chu, S. Wang, and Q. Gong, Ultrafast third-order nonlinear optical properties of graphene in aqueous solution and polyvinyl alcohol film, Chemical Physics Letters 523, 104 (2012).

[11] Q. Bao, H. Zhang, Y. Wang, Z. Ni, Y. Yan, Z. X. Shen, K. P. Loh, and D. Y. Tang, Atomic-layer craphene as a saturable absorber for ultrafast pulsed lasers, Advanced Functional Materials 19, 3077 (2009), arXiv:0910.5820.

[12] S. Kumar, M. Anija, N. Kamaraju, K. S. Vasu, K. S. Subrahmanyam, A. K. Sood, and C. N. R. Rao, Femtosecond carrier dynamics and saturable absorption in graphene suspensions, Applied Physics Letters 95, 191911 (2009), https://doi.org/10.1063/1.3264964.

[13] Z. Sun, T. Hasan, F. Torrisi, D. Popa, G. Privitera, F. Wang, F. Bonaccorso, D. M. Basko, and A. C. Ferrari, Graphene mode-locked ultrafast laser, ACS Nano 4,
803 (2010), arXiv:0909.0457

[14] G. Xing, H. Guo, X. Zhang, T. C. Sum, and C. H. A. Huan, The Physics of ultrafast saturable absorption in graphene., Optics express 18, 4564 (2010).

[15] T. B. Norris, M.-B. Lien, M. Mittendorff, M. Helm, E. Malic, T. Winzer, D. Sun, S. Winnerl, and A. Knorr, Absorption saturation in optically excited graphene, Applied Physics Letters 101, 221115 (2012)

[16] S. Omi, N. Kawaguchi, and Y. Oki, Q-switched fiber laser based on carbon nano wall saturable absorber, in Ultrafast Phenomena and Nanophotonics XIX Vol.

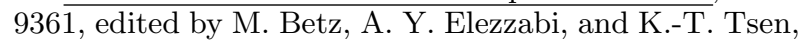
International Society for Optics and Photonics (SPIE, 2015) pp. $11-16$.

[17] G. Sobon, J. Sotor, I. Pasternak, A. Krajewska, W. Strupinski, and K. M. Abramski, Multilayer graphene-based saturable absorbers with scalable modulation depth for mode-locked er- and tm-doped fiber lasers, Opt. Mater. Express 5, 2884 (2015).

[18] K. Wang, B. M. Szydłowska, G. Wang, X. Zhang, J. J. Wang, J. J. Magan, L. Zhang, J. N. Coleman, J. Wang, and W. J. Blau, Ultrafast nonlinear excitation dynamics of black phosphorus nanosheets from visible to midinfrared, ACS Nano, ACS Nano 10, 6923 (2016)

[19] G. Demetriou, H. T. Bookey, F. Biancalana, E. Abra1606128ham, Y. Wang, W. Ji, and A. K. Kar, Nonlinear optical properties of multilayer graphene in the infrared, Opt. Express 24, 13033 (2016)

[20] S. Kurata, J. Izawa, and N. Kawaguchi, Allpolarization maintaining erbium fiber laser based on carbon nanowalls saturable absorber, in Fiber Lasers XV: Technology and Systems, Vol. 10512, edited by I. Hartl and A. L. Carter, International Society for Optics and Photonics (SPIE, 2018) pp. $490-496$.

[21] Y.-K. Choi, H.-S. Im, and K.-W. Jung, Laser ablation of graphite at $355 \mathrm{~nm}$ : cluster formation and plume propagation, International Journal of Mass Spectrometry 189, 115 (1999)

[22] K. A. Janulewicz, A. Hapiddin, D. Joseph, K. E. Geckeler, J. H. Sung, and P. V. Nickles, Nonlinear absorption and optical damage threshold of carbon-based nanostructured material embedded in a protein, Applied Physics A 117, 1811 (2014)

[23] R. Weber, M. Hafner, A. Michalowski, and T. Graf, Minimum damage in cfrp laser processing, Physics Procedia 12, 302 (2011), lasers in Manufacturing 2011 - Proceedings of the Sixth International WLT Conference on Lasers in Manufacturing.

[24] R. Yu, J. D. Cox, J. R. M. Saavedra, and F. J. García de Abajo, Analytical modeling of 
graphene plasmons, ACS Photonics 4, 3106 (2017) https://doi.org/10.1021/acsphotonics.7b00740.

[25] S. A. Mikhailov, Theory of the giant plasmon-enhanced second-harmonic generation in graphene and semiconductor two-dimensional electron systems, Phys. Rev. B 84, 045432 (2011).

[26] A. Marini, J. D. Cox, and F. J. García de Abajo, Theory of graphene saturable absorption, Phys. Rev. B 95, 125408 (2017)

[27] S. A. Mikhailov, Nonperturbative quasiclassical theory of the nonlinear electrodynamic response of graphene, Phys. Rev. B 95, 085432 (2017)

[28] K. L. Ishikawa, Nonlinear optical response of graphene in time domain, Phys. Rev. B 82, 201402 (2010).

[29] L. A. Chizhova, F. Libisch, and J. Burgdörfer, Highharmonic generation in graphene: Interband response and the harmonic cutoff, Phys. Rev. B 95, 085436 (2017)

[30] E. Malic, T. Winzer, E. Bobkin, and A. Knorr, Microscopic theory of absorption and ultrafast many-particle kinetics in graphene, Phys. Rev. B 84, 205406 (2011).

[31] Z. Zhang and P. L. Voss, Full-band quantum-dynamical theory of saturation and four-wave mixing in graphene, Opt. Lett. 36, 4569 (2011)

[32] G. Y. Guo, K. C. Chu, D.-s. Wang, and C.-g. Duan, Linear and nonlinear optical properties of carbon nanotubes from first-principles calculations, Phys. Rev. B 69, 205416 (2004).

[33] Y. Wang and D. R. Andersen, First-principles study of the terahertz third-order nonlinear response of metallic armchair graphene nanoribbons, Phys. Rev. B 93, 235430 (2016)

[34] J. J. Dean and H. M. van Driel, Graphene and few-layer graphite probed by second-harmonic generation: Theory and experiment, Phys. Rev. B 82, 125411 (2010).

[35] D. Castelló-Lurbe, H. Thienpont, and N. Vermeulen, Predicting graphene's nonlinear-optical refractive response for propagating pulses, Laser \& Photonics Reviews 14, 1900402 (2020), https://onlinelibrary.wiley.com/doi/pdf/10.1002/lpor.2019

[36] E. Runge and E. K. Gross, Density-functional theory for time-dependent systems, Physical Review Letters 52, 997 (1984).

[37] C. A. Ullrich, Time-Dependent Density-Functional Theory: (Oxford Univ $\operatorname{Pr}$ (Txt), 2012).

[38] K. Yabana and G. Bertsch, Time-dependent local-density approximation in real time, Physical Review B 54, 4484 (1996).

[39] G. F. Bertsch, J.-I. Iwata, A. Rubio, and K. Yabana, Real-space, real-time method for the dielectric function, Phys. Rev. B 62, 7998 (2000)

[40] M. Uemoto, Y. Kuwabara, S. A. Sato, and K. Yabana, Nonlinear polarization evolution using time-dependent density functional theory, The Journal of chemical physics 150, 094101 (2019).

[41] T. Otobe, High-harmonic generation in $\alpha$-quartz by electron-hole recombination, Phys. Rev. B 94, 235152 (2016)

[42] I. Floss, C. Lemell, G. Wachter, V. Smejkal, S. A. Sato, X.-M. Tong, K. Yabana, and J. Burgdörfer, Ab initio multiscale simulation of high-order harmonic generation in solids, Phys. Rev. A 97, 011401 (2018)

[43] G. Le Breton, A. Rubio, and N. Tancogne-Dejean, Highharmonic generation from few-layer hexagonal boron nitride: Evolution from monolayer to bulk response, Phys.
Rev. B 98, 165308 (2018).

[44] S. A. Sato, Y. Taniguchi, Y. Shinohara, and K. Yabana, Nonlinear electronic excitations in crystalline solids using meta-generalized gradient approximation and hybrid functional in time-dependent density functional theory, Journal of Chemical Physics 143, 10.1063/1.4937379 (2015).

[45] A. Yamada and K. Yabana, Time-dependent density functional theory for a unified description of ultrafast dynamics: pulsed light, electrons, and atoms in crystalline solids, arXiv preprint arXiv:1810.06168 (2018).

[46] M. Breusing, C. Ropers, and T. Elsaesser, Ultrafast carrier dynamics in graphite, Physical Review Letters 102, 1 (2009)

[47] M. Breusing, S. Kuehn, T. Winzer, E. Malić, F. Milde, N. Severin, J. Rabe, C. Ropers, A. Knorr, and T. Elsaesser, Ultrafast nonequilibrium carrier dynamics in a single graphene layer, Physical Review B 83, 153410 (2011).

[48] K. Yabana, T. Sugiyama, Y. Shinohara, T. Otobe, and G. Bertsch, Time-dependent density functional theory for strong electromagnetic fields in crystalline solids, Physical Review B 85, 045134 (2012).

[49] M. Lucchini, S. A. Sato, A. Ludwig, J. Herrmann, M. Volkov, L. Kasmi, Y. Shinohara, K. Yabana, L. Gallmann, and U. Keller, Attosecond dynamical franz-keldysh effect in polycrystalline diamond, Science 353, 916 (2016), https://science.sciencemag.org/content/353/6302/916.full.pdf.

[50] S. A. Sato, K. Yabana, Y. Shinohara, T. Otobe, K.-M. Lee, and G. F. Bertsch, Time-dependent density functional theory of high-intensity short-pulse laser irradiation on insulators, Phys. Rev. B 92, 205413 (2015).

[51] A. Sommer, E. M. Bothschafter, S. A. Sato, C. Jakubeit, T. Latka, O. Razskazovskaya, H. Fattahi, M. Jobst, W. Schweinberger, V. Shirvanyan, V. S. Yakovlev, R. Kienberger, K. Yabana, N. Karpowicz, M. Schultze, and F. Krausz, Attosecond nonlinear polarization and light-matter energy transfer in solids, Nature 534, 86 (2016).

[52] N. Troullier and J. L. Martins, Efficient pseudopotentials for plane-wave calculations, Physical review B 43, 1993

Conce(1199ahd Applications (Oxford Graduate Texts)

[53] J. P. Perdew and A. Zunger, Self-interaction correction to density-functional approximations for many-electron systems, Physical Review B 23, 5048 (1981).

[54] A. Castro, M. A. Marques, and A. Rubio, Propagators for the time-dependent kohn-sham equations, The Journal of chemical physics 121, 3425 (2004).

[55] M. Noda, S. A. Sato, Y. Hirokawa, M. Uemoto, T. Takeuchi, S. Yamada, A. Yamada, Y. Shinohara, M. Yamaguchi, K. Iida, et al., Salmon: Scalable ab-initio light-matter simulator for optics and nanoscience, arXiv preprint arXiv:1804.01404 (2018).

[56] E. A. Taft and H. R. Philipp, Optical properties of graphite, Physical Review 138, 10.1103/PhysRev.138.A197 (1965).

[57] H. Venghaus, Redetermination of the dielectric function of graphite, physica status solidi (b) 71, 609 (1975).

[58] F. Meng, M. D. Thomson, F. Bianco, A. Rossi, D. Convertino, A. Tredicucci, C. Coletti, and H. G. Roskos, Saturable absorption of femtosecond optical pulses in multilayer turbostratic graphene, Opt. Express 24, 15261 (2016) 
[59] H. Zhang, S. Virally, Q. Bao, L. K. Ping, S. Massar, N. Godbout, and P. Kockaert, Z-scan measurement of the nonlinear refractive index of graphene, Opt. Lett. 37, 1856 (2012). 